\title{
CONTINUOUS INCREASING FUNCTIONS OF FINITE AND
}

\section{TRANSFINITE ORDINALS*}

BY

\author{
OSWALD VEBLEN
}

\section{Introduction. $\dagger$}

A continuous increasing function of a set of ordinal numbers is analogous to a progressively continuous increasing function of the real variable. Some of its properties $\ddagger$ are developed below, especially such as bear on the notion of a derived function of the ath degree (cf. § 3) and its extensions (cf. § 4). They are nearly all generalizations of properties discovered by CANTOR $\S$ for particular functions and so may be used to simplify some of his proofs and generalize some of his results. In particular they extend his theory of $\epsilon$-numbers.

One of the most interesting problems in the theory of transfinite numbers arises in connection with HARDY's $\|$ scheme for obtaining a subset of the continuum of type $\Omega$. The success of his method depends on determining for each ordinal number $\alpha(\alpha \neq \beta+1)$ of the second class a unique fundamental sequence $S_{\alpha}=\left\{\alpha_{\nu}\right\}$ such that $L \alpha_{\nu}=\alpha$. For each number $\alpha(\alpha \neq \beta+1)$ of the second class there evidently exists an infinitude of such sequences, of which, in any special case, one may be selected. But no one has as yet given a method of determining a set of sequences $\left\{S_{a}\right\}$ such that for each $\alpha(\alpha \neq \beta+1, \omega \leqq \alpha<\Omega)$ there exists one and only one $S_{a}$.

If $\alpha$ is restricted to be less than $\epsilon_{0}$, the first $\epsilon$-number, the problem of determining $\left\{S_{a}\right\}$ is very easily solved. For every number of the second kind $\uparrow$ in the second number-class can be written uniquely in the form,***

\footnotetext{
* Presented to the Society September 5, 1907, under the title, Continuous Increasing Functions of Ordinal Numbers. Received for publication December 26, 1907.

$\dagger$ The rest of the article can be read independently of the introduction.

$\ddagger$ Many others could be transferred to this theory from the theory of the functions of a real variable, for example, the theorem that a continuous function of a continuous function is continuous.

¿qG. Cantor, Beiträge zur Begründung der transfiniten Mengenlehre, Mathematische Annalen, vol. 49 (1897).

IG. H. HARDY. Quarterly Journal of Mathematics, Vol. 35 (1903), pp. 87-94.

T Cantor, loc. cit., p. 226.

** Cantor, loc. cit., p. 237, Theorem B.
} 


$$
\alpha=\beta+\omega^{\gamma}
$$

in which $\gamma$ denotes what CANTOR calls the exponent of $\alpha$.

Now take $\{\nu\}$ ( $\nu$ finite) as the fundamental sequence for $\omega$; if $\boldsymbol{\gamma}$ is of the first kind, take $\left\{\beta+\omega^{\gamma-1} \nu\right\}$ ( $\nu$ finite) as the fundamental sequence for $\beta+\omega^{\gamma}$; and if $\boldsymbol{\gamma}$ is of the second kind, take $\left\{\beta+\omega^{\gamma_{\nu}}\right\}$ (where $\left\{\boldsymbol{\gamma}_{\nu}\right\}$ is the fundamental sequence for $\gamma$ ) as the fundamental sequence for $\beta+\omega^{\gamma}$. Since $\omega^{\alpha}>\alpha$ for all $a$ 's less than $\epsilon_{0}$, this method determines $\left\{S_{a}\right\}$ for all such $\alpha$ 's, but since $\omega^{\epsilon_{0}}=\epsilon_{0}$ it fails to determine a sequence for $\epsilon_{0}$. A method for extending this determination of $\left\{S_{a}\right\}$ much further, though not so as to include all ordinals less than $\Omega$, is given in Example 6.

$\S 1$. Let $\{x\}$ be the set of all finite and transfinite ordinal numbers less than a given ordinal $X$.

A continuous increasing function $f$ is subject to the following conditions:

1) For every $x$ of $\{x\}, f(x)$ is an ordinal number.

2) If $x_{1}<x_{2}, f\left(x_{1}\right)<f\left(x_{2}\right)$.

3) If $\left\{x^{\prime}\right\}$ is the set of all ordinals less than an ordinal $x$ of the second kind, then $f(x)$ is the least ordinal greater than every $f\left(x^{\prime}\right)$.* $^{*}$

A set of ordinals is said to be internally closed if it includes all its limitvalues with the possible exception of its least upper bound. On account of condition 3) the set of values $y$ satisfying the conditions $y=f(x)$ is internally closed. Conversely, let $\{y\}$ be any internally closed set of ordinals. Then $\{y\}$ is a well-ordered (or eutactic) set and hence is similar to the set of all ordinals less than a certain $X$. Calling the latter set $\{x\}$ and denoting the ordered correspondence between $\{x\}$ and $\{y\}$ by $f$, it is evident that the function $f$ satisfies the conditions 1$), 2), 3)$. Hence,

Theorem 1. The set of values of $f(x)$, if $f$ is any continuous increasing function, is internally closed. Conversely, any closed set of ordinals defines by its correspondence with a segment of the set of ordinals a continuous increasing function.

Example 1. As a function of $\beta, \alpha+\beta$ is continuous and increasing, but as a function of $\alpha$, it is discontinuous.

EXAMPLE 2. The product $\alpha \beta$ is a continuous increasing function of $\beta$ but not of $\alpha$.

Example 3. The numbers of the second kind of any number class and all preceding classes form an internally closed set. They therefore define a continuous increasing function $S(x)$.

* This may be expressed by the notation

$$
\underset{x^{\prime} \leq x}{\bar{B}}\left\{f\left(x^{\prime}\right)\right\}=f(x)
$$

and the language " $f(x)$ is the least upper bound of the set $\left\{f\left(x^{\prime}\right)\right\}$." 
Example 4. If $x_{a}$ is any element of a well-ordered set $\{x\}$, then the set of all elements preceding $x_{a}$ is called a section ("Abschnitt") of $\{x\}$ and the set consisting of $x_{a}$ and all following elements is called a residue ("Rest") of $\{x\} . \dagger \quad$ A well-ordered set is never similar to a section of itself, but some wellordered sets are similar to all their residues.

Such a set is called self-residual and its type or ordinal number is also called self-residual. An equivalent definition is that a self-residual number $\beta$ satisfies the equation

$$
\alpha+\beta=\beta
$$

for every $\alpha$ less than $\beta$. The smallest self-residual number is evidently $\omega$. Also if $\{\alpha\}$ is any set of self-residual numbers, $\bar{B}\{\alpha\}$ is easily seen to be selfresidual because, if it had a residue less than itself, this residue as well as the corresponding section would be less than one of the $\alpha$ 's.

Now let $X>\omega$ be the first ordinal of some number class and consider all the self-residual numbers of the set $\{x\}<X$. Since the set of self-residual numbers in $\{x\}$ has just been seen to be internally closed it defines a continuous increasing function which we shall denote by $r(x)$.

TheOREM 2. There exists one and but one continuous increasing function $f(x)$ with a given value for $f(1)$ if the value of $f(x+1)$ is given uniquely for each value of $f(x)$.

Proof. Suppose the theorem not valid; then there must be a first value $x_{0}$ for which $f(x)$ is not uniquely defined. If $x_{0}$ is of the first kind, there exists a number $x_{0}^{\prime}$ such that $x_{0}^{\prime}+1=x_{0}$ and $f\left(x_{0}^{\prime}\right)$ is uniquely defined, and therefore $f\left(x_{0}\right)$ also, contrary to the hypothesis. If $x_{0}$ is of the second kind, it is the least ordinal larger than all those smaller ordinals for which $f(x)$ is uniquely defined. Hence by Condition 3) $f\left(x_{0}\right)$ must be the least upper bound of the set of values $f(x)$ for $x<x_{0}$. Thus $f\left(x_{0}\right)$ is again uniquely defined, contrary to hypothesis.

Corollary 1. The function $S(x)$ in Example 3 above is the same as $\omega x$.

Proof. If $S(x)$ is any number of the second kind, it is well known that $S(x+1)$, the next following ordinal of the second kind, is $S(x)+\omega$. Also it is well known that

By definition,

$$
\omega \cdot(x+1)=\omega \cdot x+\omega .
$$

$$
\omega=\omega \cdot 1=S(1) \text {. }
$$

As $\omega \cdot x$ and $S(x)$ are both continuous increasing functions, Theorem 2 gives

$$
\omega \cdot x=S(x)
$$

† Cantor, loc. cit., p. 210. 
Example 5. According to Cantor's definition $†$ when translated into our terminology, $\alpha^{x}$ is the one continuous increasing function for which

$$
\alpha^{1}=\alpha, \quad \alpha^{x+1}=\alpha^{x} \cdot \alpha .
$$

Corollary 2. The self-residual function $r(x)$ defined in Example 4 is the same as $\omega^{x}$.

Proof. If $x$ is self-residual, $x+\alpha(\alpha \leqq x)$ can not be self-residual because one of its residues is $\alpha$. Hence the self-residual ordinal next larger than $x$ is $x \cdot \omega$. Since $r(1)=\omega$, this requires by Theorem 2 that

$$
r(x)=\omega^{x} \text {. }
$$

Theorem 3. A continuous increasing function $f$ always satisfies the condition

$$
f(x) \geqq x .
$$

Proof. Supposing the theorem untrue, let $x_{0}$ be the first value for which $f(x)<x$. Since $f(1) \geqq 1, x_{0}>1$. Hence if $x_{0}$ were of the first kind, $f\left(x_{0}^{\prime}\right) \geqq x_{0}^{\prime}$ where $x_{0}^{\prime}+1=x_{0}$, and so by Condition 2) $f\left(x_{0}\right) \geqq x_{0}$, which gives a contradiction. If $x_{0}$ were of the second kind, for all preceding values of $x$ we should have $f(x) \geqq x$. But if so,

$$
\underset{x<x_{0}}{\bar{B}}\{f(x)\} \geqq \underset{x<x_{0}}{\mathbb{B}}\{x\}
$$

which is, by Condition 3), a contradiction of $f\left(x_{0}\right)<x_{0}$.

$\S 2$. Let us now introduce the conditions that $X>\omega$ shall be the first ordinal of a certain number class and that the values of $f(x)$ as well as of $x$ shall be less than $X$. The latter condition is fulfilled, as may easily be shown, whenever $f(1)<X$ and the difference between $f(x)$ and $f(x+1)$ is always less than $X$. From here on, the functional symbols $f, \phi$, etc., shall be used only for continuous increasing functions satisfying these additional conditions.

Theorem 4. There exist solutions of the equation $f(x)=x$ and these solutions $\{\xi\}$ form a closed set similar to $\{x\}$.

Proof: $\ddagger$ Let $x_{1}$, be any value of $x$. Consider the sequence

$$
y_{1}=f\left(x_{1}\right), y_{2}=f\left(y_{1}\right), y_{3}=f\left(y_{2}\right), \cdots, y_{n}=f\left(y_{n-1}\right), \cdots
$$

for all finite values of $n$. The least upper bound $\xi$ of the arguments $y_{n-1}$ is also the least upper bound of the functional values, $y_{n}$. Hence, by Condition 3 ) of the definition of a continuous increasing function, $f(\xi)=\xi$. This shows that for every value of $x$ there is a larger or equal value of $\xi$. As $\{\xi\}$ is a subset of $\{x\}$ this requires that $\{\xi\}$ and $\{x\}$ shall be similar. To show that $\{\xi\}$ is closed,

† Loc. cit., p. 233.

$\ddagger$ Cf. Cantor's proof of Theorem. A, loc. cit, p. 242. 
let $\left\{\xi^{\prime}\right\}$ be any subset of $\{\xi\}$ whose least upper bound $x_{0}$ is of the second kind. Being identical with $\left\{\xi^{\prime}\right\}$, the set $\left\{f\left(\xi^{\prime}\right)\right\}$ has also the upper bound $x_{0}$. Hence $f\left(x_{0}\right)=x_{0}$ and $x_{0}$ belongs to $\{\xi\}$.

The continuous increasing function defined by the correspondence between $\{x\}$ and $\{\xi\}$ is called the first derived function of $f$.

Corollary 1. If $f^{\prime}$ is the first derived function of $f, f^{\prime}(1)$ is the least upper bound of $f(1), f[f(1)], \ldots$, and if $f^{\prime}(x)<\alpha<f^{\prime}(x+1)$, then $f^{\prime}(x+1)$ is the least upper bound of $f(\alpha), f[f(\alpha)], \cdots$

Corollary 2. The first derived function of $\alpha+x$ is $\alpha \cdot \omega+(x-1) \cdot \dagger$

Corollary 3. The first derived function of $\alpha \cdot x$ is $\alpha^{\omega} \cdot x$.

Proof. By Corollary 1, $\alpha^{\omega}$ is the first ordinal which satisfies

Also if $\xi$ satisfies (1),

$$
\alpha \cdot x=x \text {. }
$$

$$
\alpha(\xi+1)=\alpha \xi+\alpha=\xi+\alpha,
$$

and hence by Corollary $1, \xi+\alpha^{\omega}$ is the next ordinal satisfying (1). According to Theorem 2, this shows that $\alpha^{\omega} \cdot x$ is identical with the first derived function of $\alpha \cdot x$.

Corollary 4. The first derived function of $\omega^{x}$ is the function $\epsilon$, where $\epsilon(x)$ stands for the $\epsilon$-number $\epsilon_{x-1} \cdot \$$

Corollary 5. The first derived function of $\alpha^{x}(\alpha>\omega)$ is the function $f$, where $f(x)$ stands for the $\epsilon$-number $\epsilon_{\beta+(x-1)}$ if $\epsilon_{\beta}$ is the first $\epsilon$-number larger than $\alpha$. The first derived function of $n^{x}$ ( $n$ finite) is the function $\phi$, where $\phi(1)=\omega$ and $\phi(x)=\epsilon_{x-2}$ when $1<x . \S$

Theorem 5. $\|$ If $\left\{S_{a}\right\}$ is a well-ordered set of internally closed sets $S_{a}$ of ordinals, each $S_{a}$ being a subset of $\{x\}$ similar to $\{x\}$ and also a subset of each $S_{a}$ which precedes it, then, provided the cardinal number $\overline{\left\{\overline{\left.S_{a}\right\}}\right.}$ is less than $\overline{\overline{\{x\}}}$, there exists an internally closed set $S$ which includes all the ordinals common to all $S_{a}^{\prime}$ 's and is similar to $\{x\}$.

Proof. Let $x$ be any ordinal of $\{x\}$ and $y_{a}$ be the first ordinal of $S_{a}$ which is larger than $x$. The set $\left\{y_{a}\right\}$ is, by hypothesis, of cardinal number less than $\overline{\{x\}}$. Hence as $\left\{y_{a}\right\}$ is a subset of $\{x\}$ it has a least upper bound $y$ in $\{x\}$.

Since $\left\{y_{a}\right\}$ is a subset of $S_{1}, y$ is a member of $S_{1}$. In like manner, since the set of all $y_{\alpha}$ 's for which $\alpha>\beta$ is a subset of $S_{\beta}, y$ is a member of $S_{\beta}$. Hence $y$ is a member of every $S_{a}$. Thus the set $S$ of all ordinals $y$ common to the sets $S_{a}$ exists and is similar to $\{x\}$. That $S$ is internally closed follows from the fact that any subset $S^{\prime}$ of $S$ whose least upper bound is not $X$ is a subset of each $S_{a}$

$\dagger$ Here it is to be remembered that if $x>\omega, x-1=x$.

$\ddagger$ CANTOR, loc. cit., \& 20.

\& CantoR, theorems $G$ and $H$, p. 245.

|| For the notation cl. Cantor, Mathematische Annalen, vol. 46 (1895), pp. 481-2. 
and hence as each $S_{a}$ is internally closed the least upper bound of $S^{\prime}$ is a member of each $S_{a}$.

TheORem 6. For every continuous increasing function $f(x)$ there exists a well-ordered set of continuous increasing functions $f(x, \alpha), \alpha<X$, such that the values of $f(x, \alpha)$ (for fixed $\alpha$ and variable $x$ ) are the set of all ordinals which are solutions of

for every $\beta$ less than $\alpha$.

$$
f(x, \beta)=x,
$$

Proof. In view of Theorems 4 and 1 this is a corollary of Theorem 5. The first derived function of $f(x, \alpha)$ is $f(x, \alpha+1)$, and $f(x, \alpha)$ may be called the ath derived function of $f(x)$.

Corollary 1. If $f(1)>1$ the function $f(1, \alpha)$ is a continuous increasing function of $\alpha, \alpha$ taking all values of $\{x\}$.

Proof. Since $f(1)>1$, we have $f(1,1)>f(1)$ by Condition 2$)$ of the definition of a continuous increasing function taken with Corollary 1 of Theorem 4. In like manner $f(1, \alpha+1)>f(1, \alpha)$, and hence Conditions 1) and 2) of the definition of a continuous increasing function are satisfied by $f(1, \alpha)$. To show that Condition 3) is satisfied, consider any set of $\alpha$ 's, $\left\{\alpha^{\prime}\right\}$, whose least upper bound $\beta$ is not $X . \bar{B}\left\{f\left(1, \alpha^{\prime}\right)\right\}$ is a value of $f\left(x, \alpha_{0}^{\prime}\right)$ for each $\alpha_{0}^{\prime}$ of $\left\{\alpha^{\prime}\right\}$ because every value of $f\left(1, \alpha^{\prime}\right)$ for $\alpha^{\prime}>\alpha_{0}^{\prime}$ is a value of $f\left(x, \alpha_{0}^{\prime}\right)$. Hence as $f(1, \beta)$ cannot be less than $\bar{B}\left\{f\left(1, \alpha^{\prime}\right)\right\}$ it must be equal to it.

Corollary 2. If $f(x)=\gamma+x$ ( $\gamma$ constant),

$$
f(x, \alpha)=\gamma \cdot \omega^{\alpha}+(x-1),
$$

and so $\gamma \cdot \omega^{\alpha}$ is a continuous increasing function of $\alpha$.

Corollary 3. If $f(x)=\gamma \cdot x$ ( $\gamma$ constant),

$$
f(x, \alpha)=\gamma^{\omega^{a}} \cdot x,
$$

and hence the function $\gamma^{\omega^{\alpha}}$ is a continuous increasing function of $\alpha$.

Corollary 4. If $f(x)=\omega^{x}, f(x, \alpha)$ is the $(\alpha-1)$ th derived function of $\epsilon(x)$. For each $\alpha,\{f(x, \alpha)\}$ is an internally closed set of $\epsilon$-numbers. Also $\{f(1, \alpha)\}$ is an internally closed set of $\epsilon$-numbers.

$\S 3$. In order to extend the notion of a derived function still further, let us consider the set of symbols

$$
\left\{\phi\left(x_{1}, x_{2}, \cdots, x_{\beta}\right)\right\}=\{\phi\},
$$

where $\beta$ may be any ordinal less than $X$ and $x_{\alpha}\left(\alpha=1,2, \ldots, \omega_{1}, \ldots, \beta\right)$ may be any ordinal less than $X$, though in a given symbol $\phi$ only a finite number of values $x_{\alpha}$ are different from 1 . We shall refer to a symbol $\phi\left(x_{1}, x_{2}, \ldots, x_{\beta}\right)$ 
as of $\beta$ th order. Let the set $\{\phi\}$ be ordered according to last differences, $\dagger$ i. e., as follows: Of two symbols

$$
\phi_{1}=\phi\left(x_{1}, x_{2}, \cdots, x_{\beta}\right), \quad \phi_{2}=\phi\left(y_{1}, y_{2}, \cdots, y_{\gamma}\right),
$$

$\phi_{1}$ precedes $\phi_{2}$ if $\beta<\gamma$, or if $\beta=\gamma$ and $x_{\beta}<y_{\gamma}$, or if $\beta=\gamma$ and $x_{\alpha}<y_{a}$ for some $\alpha(\alpha<\beta)$ while $x_{\rho}=y_{\rho}$ for all values of $\rho$ such that $\alpha<\rho \leqq \beta$.

According to this rule $\{\phi\}$ is simply ordered and it may be shown to be well ordered by proving that every subset $\left\{\phi^{\prime}\right\}$ has a first element. Let $\beta_{1}$ be the smallest final subscript in any element of $\left\{\phi^{\prime}\right\}$. The elements of $\{\phi\}$ of order $\beta_{1}$ form a set $\left\{\phi^{\prime \prime}\right\}$ and precede all other elements of $\left\{\phi^{\prime}\right\}$. Let $y_{\beta_{1}}$ be the smallest value of $x_{\beta_{1}}$ in any element of $\left\{\phi^{\prime \prime}\right\}$.

If there is more than one element of this kind, they form a set $\left\{\phi_{1}\right\}$ of elements which precede all other elements of $\left\{\phi^{\prime}\right\}$. Let $\beta_{2}$ be the lowest subscript in any element $\bar{\phi}$ of $\left\{\phi_{1}\right\}$ such that in $\bar{\phi}$ nothing but 1's appear between $x_{\beta_{2}}$ and $y_{\beta_{1}}$, and let $y_{\beta_{2}}$ be the smallest $x_{\beta_{2}}$ in any element $\bar{\phi}$. This determines a set of elements $\left\{\phi_{2}\right\}$ which precede all other elements of $\left\{\phi^{\prime}\right\}$. If $\left\{\phi_{2}\right\}$ contains more than one element, repeat this process, obtaining sets $\left\{\phi_{3}\right\},\left\{\phi_{4}\right\}, \cdots,\left\{\phi_{n}\right\}$ where $\phi_{\kappa}$ consists of elements of the type

$$
\phi\left(x_{1}, \cdots, y_{\beta_{\kappa}}, 1_{\beta_{k}+1}, \cdots, y_{\beta_{k-1}}, 1_{\beta_{k-1}+1}, \cdots, y_{\beta_{1}}\right) .
$$

By a finite number of steps this must lead to a set $\left\{\phi_{m}\right\}$ consisting of only one element $\phi_{m}$, because a sequence $\beta_{1}, \beta_{2}, \beta_{3}, \ldots$ of type $\omega^{*}$ is impossible. Thus $\phi_{m}$ is the required first elements of $\left\{\phi^{\prime}\right\}$.

The symbols of $\{\phi\}$, aside from $\phi(1)$ the first element, fall into seven classes. $\neq$

A. $\phi\left(1_{1}, 1_{2}, \cdots, 1_{a}\right)$ ( $\alpha$ of 1 st kind)

$$
=\bar{B}\left\{\phi\left(1_{1}, 1_{2}, \ldots, x_{a^{\prime}}\right)\right\} \text { for all values of } x_{a^{\prime}} \text { where } \alpha^{\prime}+1=\alpha .
$$

B. $\phi\left(1_{1}, 1_{2}, \ldots, 1_{a}\right)$ ( $\alpha$ of $2 \mathrm{~d}$ kind $)$

$$
=\bar{B}\left\{\phi\left(1_{1}, 1_{2}, \cdots, 1_{\gamma}\right)\right\} \text { for } \gamma<\alpha .
$$

C. $\phi\left(x_{1}, \ldots, x_{\beta}\right)\left(x_{1}>1,1\right.$ st kind $)$ has a next preceding element $\phi\left(x_{1}^{\prime}, \ldots, x_{\beta}\right)$, where $x_{1}^{\prime}+1=x_{1}$.

D. $\phi\left(1_{1}, \cdots, x_{a}, \cdots, x_{\beta}\right)\left(\alpha>1,1\right.$ st kind, $x_{a}$ of 1st kind $)$

$$
=\bar{B}\left\{\phi\left(1_{1}, \cdots, x_{\alpha^{\prime}}, x_{\alpha}^{\prime}, \cdots, x_{\beta}\right)\right\}
$$

†The ordering of various sets analogous to $\{\phi\}$ according to first or last differences ha been stadied by F. HAUSDORFF; Untersuchungen über Ordnungstypen, Leipziger Berichte, 1906-07.

$\ddagger$ In this notation $x_{a}$ indicates the first non-unity ordinal in the symbol

if such an ordinal exists.

$$
\phi\left(1_{1}, \cdots, x_{\alpha}, \cdots, x_{\beta}\right)
$$


for all $x_{\alpha^{\prime}}<X$ where $x_{\alpha}^{\prime}+1=x_{\alpha}$ and $\alpha^{\prime}+1=\alpha$.

$E . \phi\left(1_{1}, \cdots, x_{a}, x_{a+1}, \cdots, x_{\beta}\right) \quad\left(\alpha \geqq 1\right.$, 1st kind, $x_{\alpha}$ of $2 \mathrm{~d}$ kind $)$

$=\bar{B}\left\{\phi\left(1_{1}, \ldots, x_{a}^{\prime}, x_{\alpha+1}, \ldots, x_{\beta}\right)\right\}$ for all $x_{\alpha}^{\prime}<x_{\alpha}$.

$F \cdot \phi\left(1_{1}, \cdots, x_{a}, \cdots, x_{\beta}\right) \quad$ ( $\alpha$ of $2 \mathrm{~d}$ kind, $x_{\alpha}$ of 1 st kind)

$=\bar{B}\left\{\phi\left(1_{1}, \ldots, 2_{\gamma}, \ldots, x_{a}^{\prime}, \ldots, x_{\beta}\right)\right\}$ for all $\gamma<\alpha$ where $x_{\alpha}^{\prime}+1=x_{\alpha}$.

G. $\phi\left(1_{1}, \ldots, x_{a}, \cdots, x_{\beta}\right) \quad$ ( $\alpha$ of $2 \mathrm{~d}$ kind, $x_{\alpha}$ of $2 \mathrm{~d}$ kind)

$=\bar{B}\left\{\phi\left(1_{1}, \cdots, x_{a}^{\prime}, \ldots, x_{\beta}\right)\right\}$ for all $x_{\alpha}^{\prime}<x_{\alpha}$.

Note that the elements of $\{\phi\}$ of the first kind are $\phi(1)$ and the class $C$. The elements of the second kind which are least upper bounds of sequences of type $X$ are classes $A$ and $D$; and those which are least upper bounds of sequences which are sections of $X$ are classes $B, E, F$, and $G$. All the elements of classes $A$ and $B$ form an internally closed set of order-type $X$. Again all symbols of type

$$
\phi\left(1_{1}, 1_{2}, \cdots, x_{a}, \cdots, x_{\beta}\right),
$$

where all the digits except $x_{a}$ are fixed, form an internally closed set. It may happen that all the elements of one internally closed set come between two elements of some other set, as

$$
\left\{\phi\left(x_{1}, 1_{2}, \cdots, x_{a}, \cdots, x_{\beta}\right)\right\}
$$

come between two elements of

$$
\left\{\phi\left(1_{1}, 1_{2}, \cdots, x_{a}, \cdots, x_{\beta}\right\} .\right.
$$

$\S 4$. Let $\phi\left(x_{1}\right)$ be any continuous increasing function, i. e., let $\left\{\phi\left(x_{1}\right)\right\}$ be an internally closed subset of $\left\{x_{1}\right\}$. Then by $\phi\left(x_{1}, 1_{2}, 1_{3}, \ldots, 1_{\beta}\right)$ is meant the set of all common solutions of the equations

$$
\phi\left(1_{1}, 1_{2}, \cdots, x_{\gamma}\right)=x_{\gamma}
$$

for $\gamma<\beta$, and by $\phi\left(x_{1}, 1_{2}, 1_{3}, \ldots, x_{a}, \ldots, x_{\beta}\right), \alpha>1$, is meant the set of all common solutions of the equations

$$
\phi\left(1_{1}, 1_{2}, \cdots, x_{\gamma}, 1_{\gamma+1}, \cdots, x_{\alpha}^{\prime}, \cdots, x_{\beta}\right)=x_{\gamma}
$$

for $\gamma<\alpha$ and $x_{\alpha}^{\prime}<x_{a}$, the numbers $x_{\rho}$ in the symbol being constants with the exception of $x_{\gamma}$ and $x_{a}^{\prime}$.

Note that this coincides with the definition of derived function in $\$ 2$ for the cases $\phi\left(x_{1}, x_{2}\right)$. As an extension of Theorem 6 , we have 
THEOREM 7. If $\phi\left(x_{1}\right)$ is an arbitrary continuous increasing function for which $\phi(1)>1$, this definition determines one and only one value of $x<X$ for each symbol $\phi\left(x_{1}, \cdots, x_{\beta}\right)$.

Proof. (a). We first show that if the theorem is true for all symbols preceding a certain one,

$$
\phi\left(1_{1}, \cdots, \bar{x}_{a}, \bar{x}_{a+1}, \cdots, \bar{x}_{\beta}\right)=\bar{\phi},
$$

and if $\phi\left(1_{1}, \ldots, x_{a}, \ldots, x_{\beta}\right)=\delta\left(x_{a}\right)$ is any function of $x_{a}$ (all ordinals before $x_{a}$ in the symbol being 1 , as usual) the symbols for all of whose values precede $\bar{\phi}$, then $\delta\left(x_{a}\right)$ is a continuous increasing function.

If this were not so, let $\phi\left(1_{1}, \ldots, x_{\gamma}, \ldots, x_{\delta}\right)$ be the first element which appeared in a function $\delta\left(x_{a}\right)$ which was not continuous and increasing. The first non-continuous increasing function in which this element could appear would be

$$
\phi\left(1_{1}, \cdots, x_{e}, \cdots, x_{\gamma}, \cdots, x_{\delta}\right)=\delta\left(x_{\epsilon}\right)
$$

Since the sets of equations (1) and (2) in the definitions are well-ordered and of cardinal less than $\{x\}$, if $\epsilon=1$, the function $\delta\left(x_{\epsilon}\right)$ would have to be continuous and increasing on account of Theorems 4 and 5 .

But if $\epsilon>1$, the definition shows that when $x_{\epsilon}$ is of the firs ${ }^{+}$kind and $x_{\epsilon}^{\prime}$ is its next preceding ordinal,

$$
\begin{array}{r}
\phi\left(1_{1}, \cdots, x_{\epsilon}^{\prime}, \cdots, x_{\gamma}, \ldots, x_{\delta}\right)<\phi\left(2_{1}, 1_{2}, \cdots, x_{\epsilon}^{\prime}, \cdots, x_{\gamma}, \cdots, x_{\delta}\right) \\
<\phi\left(1_{1}, \cdots, x_{\epsilon}, \cdots, x_{\gamma}, \cdots, x^{\delta}\right),
\end{array}
$$

and hence $\delta\left(x_{\epsilon}\right)$ satisfies Condition 2) of the definition of a continuous increasing function. The definition also shows that when $x_{\mathrm{e}}$ is of the second kind $\delta\left(x_{\epsilon}\right)=\bar{B} \delta\left(x_{\epsilon}^{\prime}\right)$ for $x_{\epsilon}^{\prime}<x_{\epsilon}$ and hence $\delta\left(x_{\epsilon}\right)$ satisfies Condition 3) as well.

Condition 1 ) is evidently satisfied. Hence $\delta\left(x_{e}\right)$ is always a continuous increasing function, contrary to hypothesis.

(b) Now supposing our theorem untrue, let $\phi\left(x_{1}, \ldots, x_{\beta}\right)=\Phi$ be the first symbol for which there is not determined a unique value. $\Phi$ cannot belong to classes $A$ or $B$ (cf. $\S 3$ ) because then the first part of our definition determines for it a value which exists and is unique by Theorems 4 and 5 together with paragraph $(a)$ of this proof. In like manner $\Phi$ cannot belong to class $C$ or to class $E$ with $\alpha=1$ because when $x_{2}=1, \ldots, x_{\beta}=1$ its value is determined by first part of our definition and in the other cases by the second part. $\Phi$ cannot belong to the classes $D, E, F$, or $G$ because in these cases its value is determined by the second part of the definition.

Corollary. $\phi\left(1_{1}, \ldots, x_{\alpha}, \ldots, x_{\beta}\right)$ is a continuous increasing function of $x_{a^{*}}$ 
Theorem 8. $\phi\left(1_{1}, \cdots, x_{a}, \ldots, x_{\gamma}, \cdots, x_{\beta}\right)>x_{\gamma}$ if $x_{a}>1$.

Proof. By Theorem. 3 and the corollary above,

and for a given $x_{\gamma}$

$$
\phi\left(1_{1}, \cdots, 1_{a}, \cdots, x_{\gamma}, \cdots, x_{\beta}\right) \geqq x_{\gamma},
$$

$$
\phi\left(1_{1}, \cdots, x_{\alpha}, \cdots, x_{\gamma}, \cdots, x_{\beta}\right)>\phi\left(1_{1}, \ldots, 1_{a}, \cdots, x_{\gamma}, \ldots, x_{\beta}\right) .
$$

Corollary. $\phi\left(1_{1}, \cdots, x_{a}, \cdots, x_{\gamma}, \cdots, x_{\beta}\right)$ is not a continuous increasing function of $x_{y}$ if $x_{a}>1$.

TheOREM 9. $\phi\left(1_{1}, \cdots, 1_{\beta}\right)$ is a continuous increasing function of $\beta$.

This is evident from the definition of this function. On the other hand $f\left(1_{1}, \ldots, 2_{\beta}\right)$ is not a continuous increasing function because on account of the inequality

$$
f\left(1_{1}, \cdots, 2_{\beta}\right)>f\left(1_{1}, \cdots, 1_{\beta}\right) \geqq \beta
$$

it cannot satisfy $f\left(1_{1}, \cdots, 2_{\beta}\right)=\beta$.

This new continuous increasing function

$$
f\left(1_{1}, \ldots, 1_{\beta}\right)=\psi(\beta)
$$

can be used to generate a new set of functions

$$
\psi\left(1_{1}, \ldots, x_{a}, \ldots, x_{\beta}\right),
$$

and the process continued indefinitely.

If from the set $S$ of functional symbols $\phi\left(x_{1}, \ldots, x_{\beta}\right)$ is omitted each symbol whose value is equal to a value of its first derived function, there remains a set of functional symbols which we denote by $T$.

Theоnem 10. The ordinal number represented by each symbol in $T$ satisfies the condition

$$
\phi\left(x_{1}, \cdots, x_{\gamma}, \cdots, x_{\beta}\right)>x_{\gamma} \quad(1 \leqq \gamma \leqq \beta) .
$$

Proof. Let $x_{\alpha}$ be the first non-unity element in $\phi\left(x_{1}, \ldots, x_{\beta}\right)$ which is now to be written $\phi\left(1_{1}, \ldots, x_{a}, \ldots, x_{\beta}\right)$. Then the equation

$$
\phi\left(1_{1}, \cdots, x_{\alpha}, \cdots, x_{\beta}\right)=x_{\alpha}
$$

is impossible because in that case $\phi\left(1_{1}, \ldots, x_{a}, \ldots, x_{\beta}\right)$ would be equal to $\phi\left(x_{1}, \ldots, 1_{a}, x_{a+1}+1, \ldots, x_{\beta}\right)$ and thus could not be a member of $T$. Hence by Theorem 3

$$
\phi\left(1, \ldots, x_{\alpha}, \ldots, x_{\beta}\right)>x_{\alpha} .
$$

Now let $x_{\delta}$ be the first non-unity element after $x_{a}$. It follows that

$$
\phi\left(1_{1}, \cdots, x_{a}, \cdots, x_{\delta}, \cdots, x_{\beta}\right)>\phi\left(1_{1}, \ldots, 1_{\alpha}, \ldots, x_{\delta}, \cdots, x_{\beta}\right),
$$

and by the argument above

$$
\phi\left(1_{1}, \cdots, 1_{a}, \cdots, x_{\delta}, \cdots, x_{\beta}\right)>x_{\delta} .
$$


As there are only a finite number of non-unity elements between $x_{\alpha}$ and $x_{\beta}$ a finite number of repetitions of this step establishes the theorem.

Example 6. Let $\phi\left(x_{1}\right)=1+x_{1}$ and form the set $S$ of functions $\phi\left(x_{1}, \ldots, x_{\beta}\right)$. Then

$$
\begin{aligned}
& \phi\left(x_{1}, 1_{2}\right)=\omega+\left(x_{1}-1\right) \\
& \phi\left(x_{1}, 2_{2}\right)=\omega^{2}+\left(x_{1}-1\right) \\
& \left.\dot{\phi}\left(\dot{x_{1}}, \dot{x_{2}}\right)=\dot{\omega^{x_{2}}}+\dot{(} \cdot \dot{x_{1}}-\dot{1}\right)
\end{aligned}
$$

Hence

$$
\phi\left(1_{1}, x_{2}\right)=\omega^{x_{2}} \text {. }
$$

$\phi\left(1_{1}, 1_{2}, 1_{3}\right)=\epsilon(1)=$ the smallest $\epsilon$-number (cf. Corollary 4, Theorem 4).

$\phi\left(x_{1}, 1_{2}, 1_{3}\right)=\epsilon\left(x_{1}\right)$, which are the $\epsilon \cdot$ numbers.

$\phi\left(1_{1}, 2_{2}, 1_{3}\right)$ is the smallest $\epsilon$-number equal to its subscript.

$\phi\left(x_{1}, x_{2}, 1_{3}\right)$ are the generalization of $\epsilon$-numbers of Corollary 4, Theorem 6.

$\phi\left(x_{1}, \cdots, x_{a}\right)$ are further generalizations of $\epsilon$-numbers. By fixing all the $x_{\rho}$ 's except one which is preceded only by 1 's we obtain an internally closed subset of $\epsilon$-numbers.

The set $T$ based on the function $1+x$ gives expressions in terms of smaller ordinals in the form:

$\phi\left(x_{1}\right)=1+x_{1}$ for ordinals greater than or equal to 1 and less than $\omega$, $\phi\left(x_{1}, 1_{2}\right)=\omega+\left(x_{1}-1\right)$ for ordinals greater than or equal to $\omega$ and less than $\omega^{2}$,

$\phi\left(x_{1}, x_{2}\right)=\omega^{x_{2}}+\left(x_{1}-1\right)$ for ordinals greater than or equal to $\omega^{x_{2}}$ and less than $\omega^{x_{2}+1}$, with the exceptions of those ordinals which satisfy the condition $\phi\left(1, x_{2}\right)=x_{2}\left[=\omega^{x_{2}}\right]$. These are expressed by means of

$\phi\left(x_{1}, 1_{2}, 1_{3}\right)$ [their ranks as $\epsilon$-numbers] unless they are values of $\phi\left(x_{1}, 2_{2}, 1_{3}\right)$

Ordinals not denoted by any previous symbol are expressed in terms of $\phi\left(1_{1}, \cdots, x_{a}, \cdots, x_{\beta}\right)$ unless they satisfy the equation $\phi\left(1, \cdots, x_{a}, \cdots, x_{\beta}\right)=x_{a}$, in which case they are expressed by means of $\phi\left(x_{1}, \cdots, x_{\alpha}+1, \ldots, x_{\beta}\right)$ or some later symbol. 
By this scheme every ordinal is expressed in terms of smaller ordinals and by means of a symbol involving subscripts smaller than itself with the exception of those numbers which satisfy the equation

$$
\phi\left(1_{1}, 1_{2}, \ldots, 1_{a}, \ldots, 1_{\beta}\right)=\beta .
$$

The solutions of this equation are clearly an internally closed set and define a continuous increasing function

$$
\mathbf{E}(x)
$$

and may be called the E-numbers. Of course this function $\mathrm{E}(x)$ may be made the basis of a new set $T^{\prime}$ and the expression of ordinals in terms of smaller ordinals continued indefinitely.

We may now solve the problem of determining for each ordinal of the second kind and less than $\mathrm{E}(1)$ a unique sequence of type $\omega$ of which it is the least upper bound. Any such ordinal is expressed in terms of smaller ordinals in the form

$$
\phi\left(x_{1}, \ldots x_{a}, \cdots, x_{\beta}\right)=\Phi
$$

which belongs to one of the classes $A-G$ of $\S 3$.

If $\Phi$ is of class $A$, its sequence is $\left\{\xi^{\nu}\right\}$ where

$$
\xi^{\prime}=\phi\left(1_{1}, 1_{2}, \ldots, 1_{a^{\prime}}\right), \ldots, \xi^{\nu}=\phi\left(1_{1}, 1_{2}, \ldots, \xi_{a^{\prime}}^{\nu-1}\right) .
$$

If $\Phi$ is of class $B$, its sequence is $\left\{\phi\left(1_{1}, 1_{2}, \ldots, \mathbf{1}_{\gamma_{\nu}}\right)\right\}$ where $\left\{\boldsymbol{\gamma}_{\nu}\right\}$ is the sequence for $a$.

If $\Phi=\phi\left(x_{1}, \cdots, x_{\beta}\right)$ is of class $C$, let $\phi\left(x_{1}^{\prime}, \ldots, x_{\beta}\right)=\rho$ where $x_{1}^{\prime}+1=x$, and let $\Phi^{\prime}$ be the element obtained by replacing $x_{1}$ by 1 in $\Phi$. The element $\Phi^{\prime}$ must belong to one of the classes $A, B, D, E, F, G$. If $\Phi^{\prime}$ belongs to class $A$, then the sequence for $\Phi$ is $\left\{\xi^{\nu}\right\}$ where

$$
\xi^{\prime}=\rho+1, \ldots, \xi^{\nu}=\phi\left(1_{1}, 1_{2}, \ldots, \xi_{a^{\prime}}^{\nu-1}\right), \ldots
$$

If $\Phi^{\prime}$ belongs to class $B$, then the sequence for $\Phi$ is $\left\{\phi\left[1_{1}, 1_{2}, \ldots,(\rho+1)_{\gamma_{\nu}}\right]\right\}$ where $\left\{\boldsymbol{\gamma}_{\nu}\right\}$ is the sequence for $\alpha$.

If $\Phi^{\prime}$ belongs to class $D$, then the sequence for $\Phi$ is $\left\{\xi^{\nu}\right\}$ where $\xi^{\prime}=\rho+1, \ldots, \xi^{\nu}=\phi\left(1_{1}, \ldots, \xi_{a^{\prime}}^{\nu-1}, x_{a}^{\prime}, \ldots, x_{\beta}\right), \ldots$

If $\Phi^{\prime}$ belongs to class $E$ or $G$ then the sequence for $\Phi$ is $\left\{\phi\left[(\rho+1)_{1}, \ldots, x_{a}^{\nu}, \cdots, x_{\beta}\right]\right\}$ where $\left\{x_{a}^{\nu}\right\}$ is the sequence for $x_{a}$.

If $\Phi^{\prime}$ belongs to class $F$, then the sequence for $\Phi$ is

$$
\left\{\phi\left[(\rho+1)_{1}, \cdots, 2_{\gamma_{\nu}}, \cdots, x_{a}^{\prime}, \cdots, x_{\beta}\right]\right\}
$$

where $\left\{\boldsymbol{\gamma}_{\nu}\right\}$ is the sequence for $\alpha$. 
If $\Phi$ is of classes $D, E, F$ or $G$, unless it is of class $E$ with $\alpha=1$, its sequence is the same as the corresponding one developed above for class $C$, on setting $\rho=0$.

If $\Phi$ is of class $E$ with $\alpha=1$, its sequence is $\left\{\phi\left(x_{1}^{\nu}, \ldots, x_{\beta}\right)\right\}$ where $\left\{x_{1}^{\nu}\right\}$ is the sequence for $x_{1}=x_{a}$.

This scheme serves to define a unique sequence for each ordinal smaller than $\mathrm{E}(1)$, but it would not serve for $\mathrm{E}(1)$ because the symbol for $\mathrm{E}(1)$ belongs to class $B$ and involves a subscript equal to $\mathrm{E}(1)$ and therefore the sequence for $E(1)$ would be defined in terms of $E(1)$ itself. Of course the following procedure is possible. Let

$$
\phi\left(1_{1}, 1_{2}, \ldots, 1_{\beta}\right)=f(\beta)
$$

and form the set of functions

$$
f\left(\beta_{1}, \cdots, \beta_{a}\right)
$$

and determine sequences for all the $E$-numbers less than the first solution of

by means of the function

$$
f\left(1_{1}, \cdots, 1_{a}\right)=\alpha
$$

$$
f\left(\beta_{1}, \ldots, \beta_{a}\right),
$$

and this may be repeated indefinitely.

Princeton, N. J. 